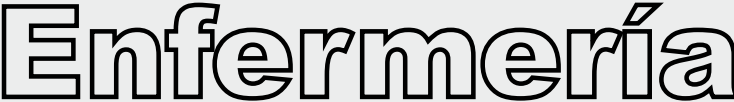 \\ Universitaria

\section{ÍNDICE ALFABÉTICO}

A

Aguilar Hernández Rosa Ma. (2):

Aguilar Ruíz Evelyn Catalina (4):

Alvarado Pacheco Paula (3):

B

Balam Gómez Maricela (4):

Balan Gleaves Cristina (1):

Balcázar Martínez Verónica (1):

Balseiro Almario Carmen Lastenia (1):

Barrientos Sánchez Judith (4):

C

Camacho Villarreal Ana Laura (1):

Camaño Vicente Elizabeth Citlali (4):

Cancino Morales Karla (1):

Cao Torija María José (3):

Carbonero Martín Miguel Ángel (4):

Cárdenas Jiménez Margarita (3):

Castro Alija María José (3):

Cruz Jiménez Maricela (3):

D

Durán Badillo Tirso (2):

G

Gallegos Torres Ruth Magdalena (1):

García Cardona Mercedes (1):

González Hernando Carolina (3):

González Hernando Carolina (4):

Grajales Alonso Ignacio (1):

Guerra Ramírez Merilyn (3):

Gutiérrez Agudelo María del Carmen (2):

Gutiérrez Sánchez Gustavo (2):

\section{H}

Hernández Cantoral Alicia (2):

Hernández Cantoral Alicia (4):

Hernández Conesa Juana María (4):

Hernández Corral Sandra (3):

Hernández Corral Sandra (4):

Hernández Zavala Margarita (4):

Hidalgo Rivera Jazmín Liliana (3):
J

Jiménez Utrilla Jorge (2):

L

Lara Ortega Fernando (4):

120

M

Maldonado Suárez Enrique (4):

133

Mantilla Pastrana María Inés (2):

Martín Villamor Pedro (3): $\quad 98$

Martín Villamor Pedro (4): $\quad 120$

Martínez Aguilar Ma. de la Luz(2): $\quad 36$

Matus Miranda Reyna (1):14; (1): 27

Mondragón Gómez Susana (2): 58

Müggenburg y Rodríguez Vigil Cristina (4): 113

Muñoz Alejandra (2):

Muñoz de Rodríguez Lucy (3):

$\mathbf{N}$

Nava Galán María Guadalupe (3):

0

Orozco González Miguel Ángel (1): 8

Ortiz Arrazola Lisueth Gladys (1): 3

\section{$P$}

8 Peña Julio (2): 50

14 Pérez Cabrera Iñiga (2): 33

98 Pérez López Juan Carlos (1): $\quad 21$

120 Piña Jiménez Irma (3): 75

3

$84 \quad \mathrm{R}$

43 Ramírez Gutiérrez Nohemí (4): 139

36 Reyes Aguilar Adelaida (1): 3

Reyes Rocha Blanca Lilia (1): $\quad 8$

Rodríguez Jiménez María Sofía (3): $\quad 92$

Rodríguez Ríos Teodomira (2): $\quad 36$

Rodríguez Sandoval Norma Aidee (2): $\quad 67$

S

Sabido Barrera Janira Guadalupe (4): $\quad 125$

Sellán Soto Carmen (4):

Sierra Aguillón Ricardo (1):

(1)

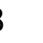

36

27

8

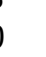

4

73

(1)

3

(1)

33

5

9

3

8

2


Solorzano García Elvira (2):

Souza de Almeida Millani (3):

T

Tello Sánchez Gamaliel Oswaldo (1):

Tenahua Quitl Inés (1):

U

Uch Pucc Pedro Fernely (4):

Uicab Pool Gloria (4):

Urra Eugenia (2):
$67 \quad \mathrm{~V}$

98 Vázquez Galindo Laura (2):

36

$\mathrm{X}$

8 Xeque Morales Ángel Salvador (1):

8

Z

Zamora Mendoza Aurora (1):

8

125 Zárate Grajales Rosa Amarilis (1):

125 Zarza Arizmendi María Dolores (1): 